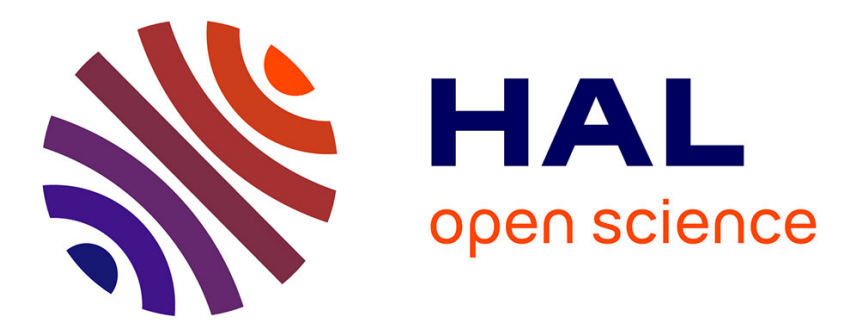

\title{
A global method for handling fluorescence spectra at high concentration derived from the competition between emission and absorption of colloidal CdTe quantum dots
}

Thomas Noblet, Laurent Dreesen, Julie Hottechamps, Christophe Humbert

\section{To cite this version:}

Thomas Noblet, Laurent Dreesen, Julie Hottechamps, Christophe Humbert. A global method for handling fluorescence spectra at high concentration derived from the competition between emission and absorption of colloidal CdTe quantum dots. Physical Chemistry Chemical Physics, 2017, 19 (39), pp.26559-26565. 10.1039/C7CP03484A . hal-02132704

\author{
HAL Id: hal-02132704 \\ https://hal.science/hal-02132704
}

Submitted on 18 Nov 2020

HAL is a multi-disciplinary open access archive for the deposit and dissemination of scientific research documents, whether they are published or not. The documents may come from teaching and research institutions in France or abroad, or from public or private research centers.
L'archive ouverte pluridisciplinaire HAL, est destinée au dépôt et à la diffusion de documents scientifiques de niveau recherche, publiés ou non, émanant des établissements d'enseignement et de recherche français ou étrangers, des laboratoires publics ou privés. 


\title{
Journal Name
}

\section{A global method for handling fluorescence spectra at high concentration derived from the competition between emission and absorption of colloidal CdTe quantum dots ${ }^{\dagger}$}

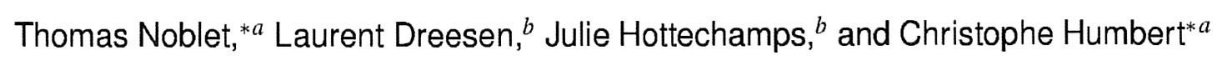

Received Date
Accepted Date

DOI: $10.1039 / \operatorname{xxxxxxxxxx}$

www.rsc.org/journalname

\begin{abstract}
We investigate the effects of CdTe quantum dot (QD) concentration on their fluorescence in water. The emission spectra, acquired in right angle geometry, exhibit highly variable shapes. The measurements evidence a critical value of the concentration beyond which the intensity and the spectral bandwidth decrease and the fluorescence maximum redshifts. To account for these observations, we develop a model based on the primary and secondary inner filter effects. The accuracy of the model ensures that the concentration dependent behaviour of QDs fluorescence is completely due to inner filter effects. This result is all the more interesting that it precludes the assumption of dynamic quenching. As a matter of fact, the decreasing of the emission intensity is not a consequence of collisional quenching but an effect of competition between fluorescence and absorption. We even show that this phenomenon is linked not only to the QD concentration but also to the geometric configuration of the setup. Hence, our analytical model can be easily adapted to every fluorescence spectroscopy configuration to quantitatively predict or correct inner filter effects in the case of QDs or any fluorophore, and thus improve the handling of fluorescence spectroscopy for highly concentrated solutions.
\end{abstract}

\section{Introduction}

Colloidal semiconductor nanocrystals (NCs), also known as quantum dots (QDs), are highly tunable materials that undoubtedly constitute one of the most functional zero-dimension systems, characterized by atomic-like carrier densities of states ${ }^{1-3}$. Their remarkable size-dependent electronic and optical properties, added to their chemical agility, give rise to new fields of application, such as light sources ${ }^{4-6}$, nonlinear optics 7,8 , photovoltaics $^{9}$, opto-electronics ${ }^{10}$, fluorescence spectroscopy ${ }^{11}$, biosensors $^{12-14}$ and biomedical imaging ${ }^{15-17}$. As fluorescent tags, QDs are especially preferred over common molecular chromophores for their greater robustness, stronger dipole moment,

\footnotetext{
a Univ Paris-Sud, Université Paris-Saclay, Laboratoire de Chimie Physique, CNRS, Bâtiment 201P2, 91405 Orsay, France.

${ }^{b}$ GRASP-Biophotonics, CESAM, University of Liege, Institute of Physics, Allée du 6 août 17, 4000 Liège, Belgium.

$\dagger$ Electronic Supplementary Information (ESI) available: Details of HR-TEM images and analysis, additional UV-vis and fluorescence spectra, calculations of concentrations and densities and full description of the method for handling fluorescence spectra. See DOI: 10.1039/b000000x/
}

higher quantum yield and longer photoluminescence decay time. As biosensors, the interest of their smallness lies in the tremendous surface-to-volume ratio that turns QDs into probes drastically sensitive to their surrounding environment. This has spurred numerous groups to examine the effect of composition, size, shape and ligand conjugation, in order to tune the properties of QDs at will, and thus allow the detection of specific molecular species ${ }^{18}$.

Several semiconductor alloys have been intensively studied, such as $\mathrm{PbS}, \mathrm{PbSe}^{19}, \mathrm{CdS}^{20}, \mathrm{CdSe}^{8,21,22}$ and $\mathrm{CdTe}^{23-25}$. First, amongst the II-VI compounds, the advantage of bulk CdTe comes from its band gap located around $1.6 \mathrm{eV}$ (i.e. $775 \mathrm{~nm}$ ) that allows thereafter tuning the energy levels of NCs in the visible spectral range. Indeed, the strong confinement of electrons and holes in CdTe QDs (whose diameters are less than $10 \mathrm{~nm}$ ) translates into the increase of the NCs band gap ${ }^{23}$. Second, CdTe QDs are chemically versatile so that their surface conjugation with molecular ligands make them water-soluble ${ }^{26}$.

To date, many groups have focused their works on changing the composition, the size and the surface passivation of QDs in 
order to study the tuning of their intrinsic properties. In the present article, we investigate the effect of the concentration of $\mathrm{CdTe} Q \mathrm{QDs}$ in water, and hence examine their interplay in aqueous phase. Whether the knowledge of the concentration of QDs is critical for all the applications stated above, understanding the concentration dependence of their properties is also of fundamental interest. For instance, it has been evidenced that the fluorescence emission of QDs decreases and redshifts at high concentration $^{27,28}$. In this paper, we explain these observations with an analytical model based on inner filter effects, optical phenomena occurring at high concentration when the chromophores hinder the penetration of the excitation beam into the solution and absorb the fluorescence emission of their neighbours ${ }^{29,30}$. In the light of the sole Beer-Lambert law, we show for the first time how critical the competition between fluorescence and absorption is, in comparison with collisional quenching that would consist in the alteration of the quantum efficiency due to additional nonradiative decay paths ${ }^{31}$.

In order to reach that goal, we first perform HR-TEM and UVvisible measurements to characterize the structural properties of CdTe NCs, and then quantitatively deduce their densities and extinction cross sections. These data prove useful to model afterwards the concentration dependent fluorescence of QDs with inner filter effects. We examine two different sizes of NCs in order to ensure that the results remain valid whether the excitation beam coincides or not with the first exciton absorption peak.

\section{Materials and methods}

\subsection{Colloidal CdTe QDs preparation}

CdTe QDs were purchased from Sigma-Aldrich: Ref. $777935 \mathrm{CdTe}$ core-type $25 \mathrm{mg}$ and Ref. $777951 \mathrm{CdTe}$ core-type $25 \mathrm{mg}$, respectively named QD520 and QD610 throughout the article given the locations of their fluorescence bands at $520 \mathrm{~nm}$ and $610 \mathrm{~nm}$ (as announced by the manufacturer) ${ }^{32,33}$. These commercial $\mathrm{CdTe}$ QDs exhibit carboxylic acid functional groups $(\mathrm{COOH})$ and are provided through water-soluble powders. The stock solutions (3.2 $10^{-4} \mathrm{M}$ and $1.310^{-4} \mathrm{M}$, respectively) and the serial dilutions were prepared in Milli-Q water (18 M $\Omega \cdot \mathrm{cm}$, Millipore, France). The concentrations of the two stock solutions correspond to the solubility limits of QD520 and QD610, respectively. To determine these maximum concentrations, we gradually added water until the QD powders completely dissolve. We then studied the highest possible concentrations of CdTe QDs in water.

\subsection{HR-TEM}

HR-TEM imaging was performed using an FEI instrument (Technai S-Twin, operating at $200 \mathrm{kV}$ ) with a JEOL $100 \mathrm{CXII}$ transmission electron microscope at an accelerating voltage of $100 \mathrm{kV}$. Drops of colloidal QDs were deposited on carbon coated copper grids. The images were processed and analyzed with ImageJ, as detailed in $\mathrm{ESI}^{\dagger}$.

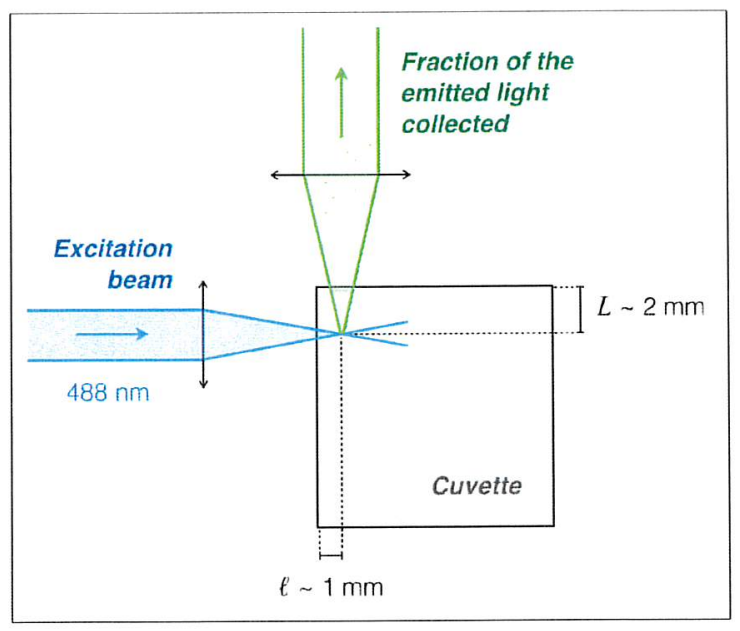

Fig. 1 Sketch of the right angle configuration used for fluorescence spectroscopy (with a $1 \mathrm{~cm} \times 1 \mathrm{~cm}$ cuvette).

\subsection{UV-visible spectroscopy}

UV-visible absorbance spectra of the colloidal solutions were recorded with a Cary-5000 spectrophotometer (Agilent) in transmission through 1-cm-length PMMA cuvettes. Milli-Q water was used as a reference so that each absorbance curve gives the signal of the colloidal CdTe QDs by differential measurements.

\subsection{Fluorescence spectroscopy}

Fluorescence spectra were acquired using an IHR-320 spectrofluorometer (Horiba) coupled with an SR-830 lock-in amplifier (Stanford Research System). Colloidal CdTe QDs were excited at $488 \mathrm{~nm}$ with a Cobolt Laser and fluorescence emission was collected in conventional right angle geometry (Figure 1). We used the same PMMA cuvettes as those for UV-visible spectroscopy.

\section{Results and discussion}

\subsection{Structural properties of CdTe QDs}

HR-TEM analysis of CdTe NCs is presented in Figure 2. Electron diffraction through NCs gives access to the pattern of their reticular planes. Measuring the size of each diffraction pattern, we deduce the size distribution of the two populations of QDs. The average diameter is $3.4+0.6 \mathrm{~nm}$ for QD520 and $3.8+0.7 \mathrm{~nm}$ for QD610 (details of the processing are given in $\mathrm{ESI}^{\dagger}$ ). As expected, the mean diameter of QD520 is smaller than that of QD610. Indeed, the energy $E_{\mathrm{QD}}$ of the first exciton absorption peak is classically related to the radius $R$ by Equation (1) ${ }^{23}$ :

$$
E_{\mathrm{QD}}=E_{g}+\frac{\hbar^{2} \pi^{2}}{2 \mu R^{2}}-\frac{1.786 e^{2}}{4 \pi \varepsilon_{0} R}-0.248 \mathscr{R},
$$

where $E_{g}=1.61 \mathrm{eV}$ is the band gap energy of bulk CdTe (at $4 \mathrm{~K}$ ), $\mu=0.077 m_{0}$ is the reduced mass of excitons (with $m_{0}$ the electron mass) and $\mathscr{R}=10 \mathrm{meV}$ is the exciton Rydberg energy. The term in $1 / R^{2}$ is the confinement energy, while that in $1 / R$ corresponds to the Coulomb attraction between the electron and the 

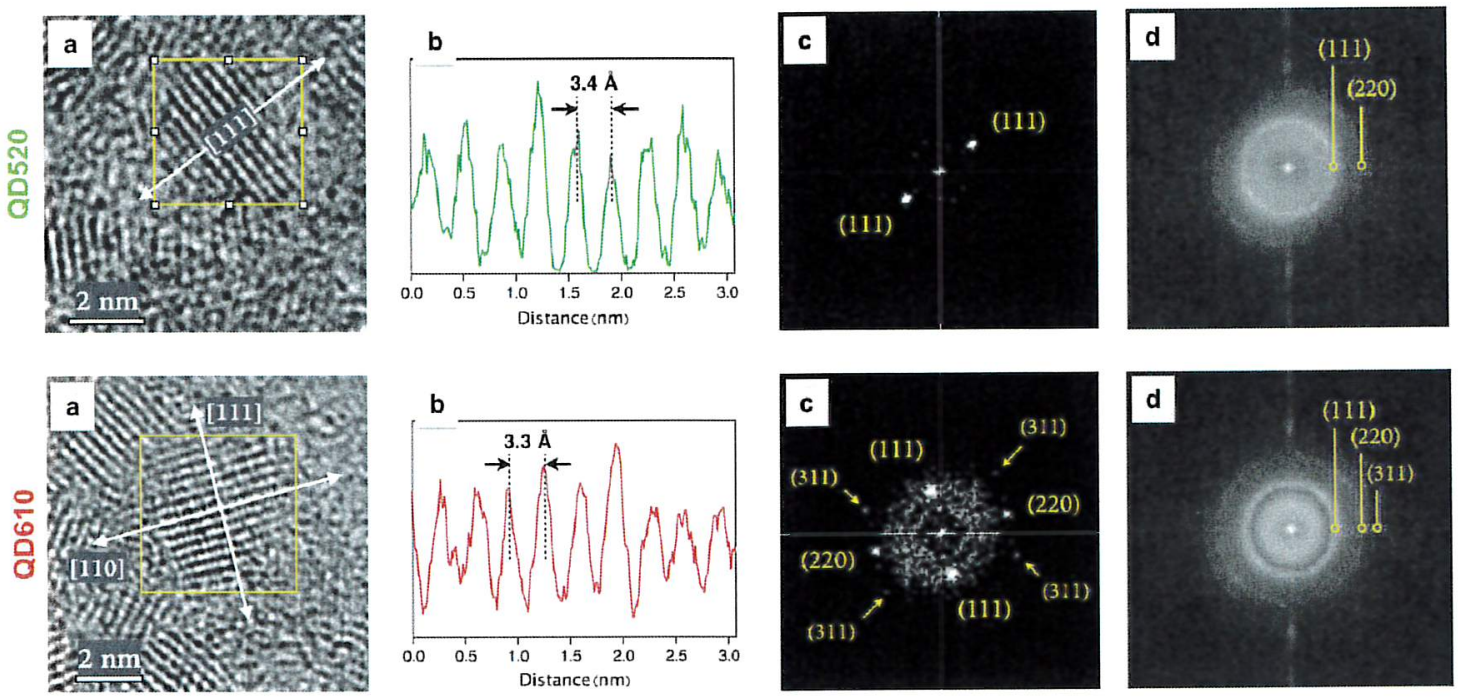

Fig. 2 (a) HR-TEM images of QD520 and QD610. The directed lines [uvw] are perpendicular to their associated reticular planes. (b) Profiles of lattice fringes along directed lines [111]. (c) FFT of unique NCs computed from the areas delimited by the yellow squares on Fig. 2a. (d) FFT of the whole HR-TEM images (available in ESI ${ }^{\dagger}$ ).

hole. The last term eventually describes their spatial correlation. This equation derives from the effective mass approximation and has already proved improper to quantitatively account for experiment $^{34-36}$. As depicted in Figure 3a, our measurements confirm that Equation (1) is not satisfactory. Moreover, whether the results we obtained for QD610 are in agreement with Ref. 34 and 35 , those of QD520 are not. Hence, it must be acknowledged that the understanding of the size dependence of the QDs properties is still incomplete: the different theoretical and empirical laws which are given in the literature cannot be generalized. This deviation may be imputable to surface properties (facets, vacancies, ligand conjugation, constraints) and lattice defects, all the more critical that the NCs are small. As a consequence, we cannot rigorously deduce the size of NCs with the sole measurement of the first exciton absorption peak.

Besides the direct measurement of the NC size, HR-TEM analysis allows calculating the interplanar spacings $d_{l k k l}$ for each family $(h k l)$ of reticular planes. Thanks to fast Fourier transforms (FFT) computed both for unique NCs (Figure 2c) and over a tenth of NCs (Figure 2d), we identified the three common diffraction peaks which correspond to the cubic zinc-blende crystal structure of CdTe thin films ${ }^{37}$. Figure $3 \mathrm{~b}$ sums up the results. The values of $d_{h k l}$, whose calculations are detailed in $\mathrm{ESI}^{\dagger}$, are quite smaller than that of bulk CdTe. For instance, we find $d_{111}=3.3 \AA$ in average whereas $d_{111}=3.7 \AA$ in the bulk ${ }^{37}$. It means that the coating of these commercial CdTe QDs exerts surface constrains on the lattice. Such small values have already been observed in the case of 4-nm core-type CdTe NCs, for which $d_{111}=3.2 \AA^{38}$. Since the $d$-spacings linearly depend on the lattice constant $a$ according to:

$$
d_{h k l}=\frac{a}{\sqrt{h^{2}+k^{2}+l^{2}}},
$$

the linear regression drawn in Figure $3 \mathrm{~b}$ enables us to deduce the lattice parameter $a=5.86 \AA$ (with a standard deviation of \pm 0.04
$\AA$ ). As explained in $\mathrm{ESI}^{\dagger}$, the knowledge of the lattice constant and the diameter of NCs allows converting mass concentrations $(\mathrm{g} / \mathrm{L})$ of $\mathrm{CdTe}$ (deduced from manufacturer data) into molar concentrations $(\mathrm{M})$ and densities $\left(\mathrm{m}^{-3}\right)$ of QDs. These data, given in Table 1, are necessary to process absorbance measurements and compute the extinction cross section of QDs.

Table 1 Concentrations of the two prepared stock solutions. The calculations are explained in $\mathrm{ESI}^{\dagger}$.

\begin{tabular}{llll}
\hline & $\begin{array}{l}\text { CdTe mass } \\
\text { concentration }(\mathrm{g} / \mathrm{L})\end{array}$ & $\begin{array}{l}\text { QD molar } \\
\text { concentration }(\mathrm{M})\end{array}$ & $\begin{array}{l}\text { QD } \\
\text { density }\left(\mathrm{m}^{-3}\right)\end{array}$ \\
\hline QD520 & 31.2 & $3.210^{-4}$ & $1.910^{23}$ \\
QD610 & 17.8 & $1.310^{-4}$ & $7.810^{22}$
\end{tabular}

\subsection{Extinction cross section of CdTe QDs}

UV-visible spectra, presented in Figure 3c, evidence that the first exciton absorption peak is located at $488 \mathrm{~nm}$ for QD520 and 566 $\mathrm{nm}$ for QD610. The former are thus resonant with the excitation wavelength $\lambda_{e x}=488 \mathrm{~nm}$ used for fluorescence measurements, while the latter are not.

In order to explain the concentration dependence of fluorescence spectra, we need to quantify the extinction cross section $\sigma\left(\lambda_{e x}\right)$ of NCs at $488 \mathrm{~nm}$. The Beer-Lambert law reads:

$$
\mathscr{A}_{N}\left(\lambda_{e x}\right)=\frac{\sigma\left(\lambda_{e x}\right) N \xi}{\ln 10}=\tilde{\sigma}\left(\lambda_{e x}\right) N \xi,
$$

where $N$ is the QD density, $\xi=1 \mathrm{~cm}$ the length of the cuvette used for UV-visible spectroscopy and $\mathscr{A}_{N}\left(\lambda_{e x}\right)$ the corresponding absorbance. For simplicity, we include the factor $\ln 10$ into the quantity $\tilde{\sigma}\left(\lambda_{e x}\right)=\sigma\left(\lambda_{e x}\right) / \ln 10$. In virtue of Equation (3), the knowledge of the QD densities henceforth allows extracting $\sigma\left(\lambda_{e x}\right)$ from the UV-visible measurements realized for successively diluted colloidal solutions, thanks to linear regressions of $\mathscr{A}_{N}\left(\lambda_{e x}\right)$ 

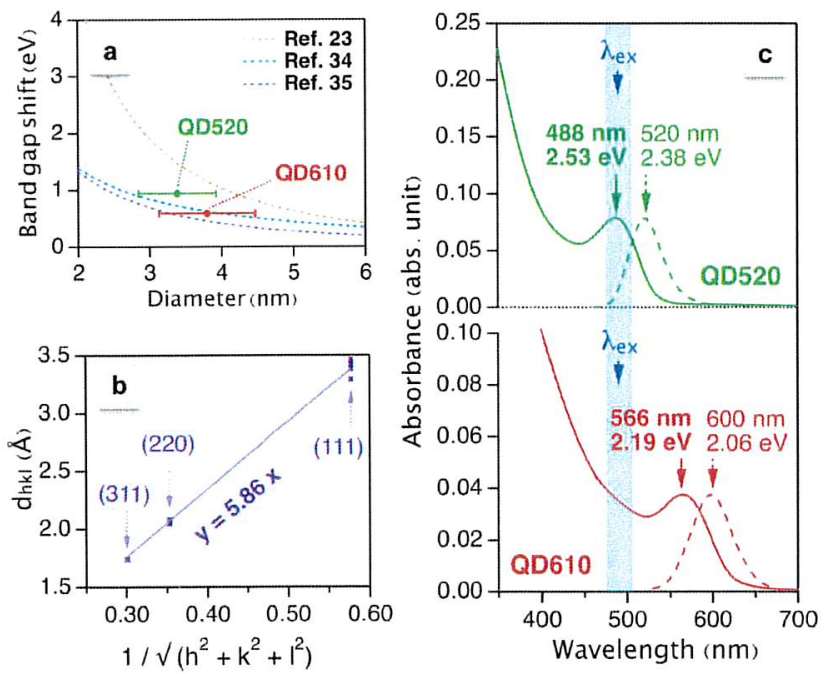

Fig. 3 (a) Band gap shift $\left(E_{\mathrm{OD}}-E_{g}\right)$ as a function of NC diameter. The green and red points correspond to our measurements and dashed lines to theoretical and empirical laws extracted from Ref. 23, 34 and 35. For QD520 and QD610, $E_{\mathrm{QD}}$ is deduced from their first exciton absorption peaks (Fig. 3c). (b) Linear regression of the interplanar spacing $y=d_{l i k l}$ with respect to $x=1 / \sqrt{h^{2}+k^{2}+l^{2}}$. The data are detailed in ESI ${ }^{\dagger}$, Table A. (c) UV-visible absorption spectra of QD520 at $3.6610^{20} \mathrm{~m}^{-3}$ and QD610 at $1.4710^{20} \mathrm{~m}^{-3}$. The dashed lines correspond to the associated fluorescence spectra (respectively normalized at the first absorption peak). The blue column indicates the excitation wavelength $\lambda_{e x}=488 \mathrm{~nm}$.

with respect to $N$. The extinction cross sections $\sigma\left(\lambda_{e x}\right)$ are then $(5.01 \pm 0.02) 10^{-20} \mathrm{~m}^{2}$ for QD520 and $(5.18 \pm 0.03) 10^{-20} \mathrm{~m}^{2}$ for QD610 (data and linear regressions are featured in $\mathrm{ESI}^{\dagger}$ ).

\subsection{Concentration dependence of fluorescence}

As depicted in Figures 4 and 5a, fluorescence spectra exhibit a significant concentration dependent shape (additional spectra are available in $\mathrm{ESI}^{\dagger}$, Figure C1). Drawing the intensity (Figure 4a), the wavelength $\lambda_{f}^{\max }$ of maximum emission (Figure 4b), and the spectral bandwidth (Figure 4c) with respect to the QD density, we evidence a critical density $N^{*} \sim 10^{22} \mathrm{~m}^{-3}$ (i.e. $\sim 20 \mu \mathrm{M}$ ) which delimits two regimes: first, for $N<N^{*}$, the fluorescence intensity increases with the density whereas the bandwidth and $\lambda_{f}^{\max }$ are quite constant; second, for $N>N^{*}$, the fluorescence intensity and the bandwidth decrease while $\lambda_{f}^{\max }$ redshifts. Especially, the behaviour of $\lambda_{f}^{\max }$ can be fitted by piecewise functions of the form:

$$
y(x)=\left\{\begin{array}{cl}
c & \text { if } x \leqslant N^{*} \\
a \log x+b & \text { if } x>N^{*}
\end{array},\right.
$$

where $a, b$ and $c$ are fitting parameters. For QD520 and QD610, we respectively determine:

$$
\lambda_{f}^{\max }(N)=\left\{\begin{array}{cc}
521 & \text { if } N \leqslant 910^{21} \mathrm{~m}^{-3} \\
23.11 \log N+13.74 & \text { if } N>910^{21} \mathrm{~m}^{-3}
\end{array},\right.
$$
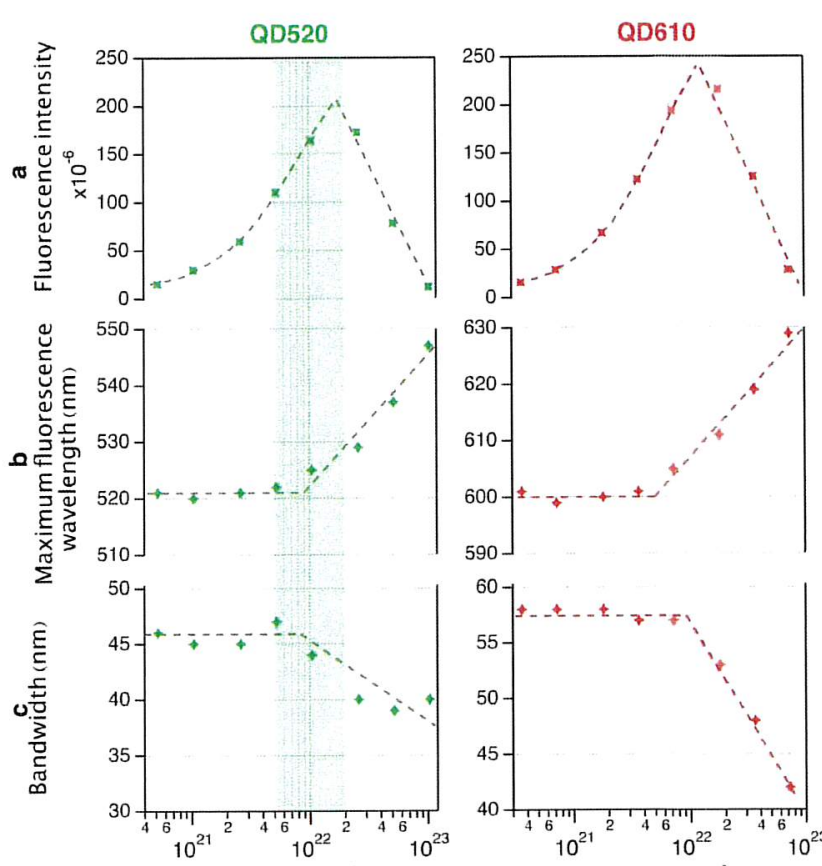

Density $\left(\mathrm{m}^{-3}\right)$

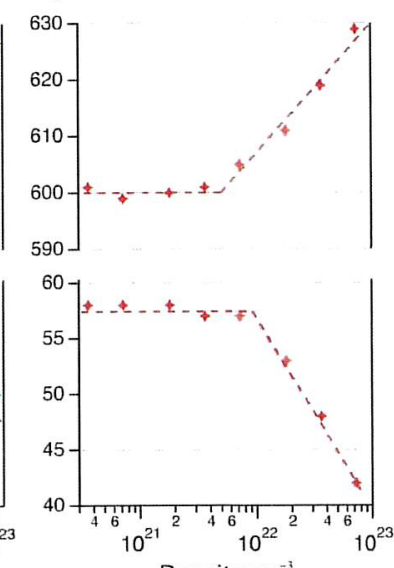

Fig. 4 (a) Maximum of fluorescence intensity with respect to QD density (b) Location of the maximum of fluorescence with respect to $Q D$ density. (c) Full width at half maximum of fluorescence spectra with respect to QD density. The dashed lines in (a) and (c) are drawn to guide the eye while those in (b) are fits to the experimental data, whose equations are given in the form of Eq. (4). The colored areas locate the critical regime. (The fluorescence spectra from which these measurements are directly extracted all feature in $\mathrm{ESI}^{\dagger}$, Figure $\mathrm{C} 1$.)

and:

$$
\lambda_{f}^{\max }(N)=\left\{\begin{array}{cc}
600 & \text { if } N \leqslant 510^{21} \mathrm{~m}^{-3} \\
23.08 \log N+99.16 & \text { if } N>510^{21} \mathrm{~m}^{-3}
\end{array}\right.
$$

In particular, we can notice that the fluorescence band of QD610 at low concentration is located at $600 \mathrm{~nm}$, contrary to the $610 \mathrm{~nm}$ announced by the manufacturer ${ }^{33}$.

Although this behaviour has already been observed for $\mathrm{CdTe}$ $\mathrm{NCs}^{28}$, the inner filter effects have never been used to give a full and quantitative account of such a concentration dependence. In the following, we describe and implement these effects and show they completely account for the observations.

\subsection{Implementing inner filter effects}

As sketched up in Figure 1, the excitation light beam which illuminates the colloidal solutions of CdTe QDs is focused in the cuvette after a pathlength $\ell \sim 1 \mathrm{~mm}$. The intensity is partly absorbed throughout this pathlength so that the fluorescence induced by the excitation beam for each QD decreases when the concentration of QDs increases. Here manifests the primary inner filter effect (PIFE) as a result of the optical absorption of the QDs in the excitation wavelength range ${ }^{30}$. Moreover, after it is emitted, the fluorescence can be re-absorbed. This secondary inner filter 
effect (SIFE), due to the QDs absorption in the emission range, implies an additional decrease of the fluorescence intensity ${ }^{30}$.

In order to model these two inner filter effects, we first express the excitation beam intensity $I\left(\lambda_{e x}\right)$ at the observed point, i.e. after a pathlength of $\ell$, thanks to the Beer-Lambert law:

$$
I\left(\lambda_{e x}\right)=I_{0} 10^{-\tilde{\sigma}\left(\lambda_{e x}\right) N \ell},
$$

where $I_{0}$ is the incident beam intensity. The fluorescence intensity $F_{N}^{\prime}\left(\lambda_{f}\right)$ at the emission wavelength $\lambda_{f}$ is then proportional to (i) the excitation beam intensity $I\left(\lambda_{e x}\right)$ at the observed point, (ii) the absorption cross section $\sigma_{\mathrm{abs}}\left(\lambda_{e x}\right)$, (iii) the fluorescence quantum yield $\phi_{f}$, and (iv) the density $N$ of QDs:

$$
F_{N}^{\prime}\left(\lambda_{f}\right) \propto I\left(\lambda_{e x}\right) \sigma_{\mathrm{abs}}\left(\lambda_{e x}\right) \phi_{f} N .
$$

Hence, the PIFE can be modeled by the following equation:

$$
F_{N}^{\prime}\left(\lambda_{f}\right)=f_{0}\left(\lambda_{e x}\right) N 10^{-\tilde{\sigma}\left(\lambda_{e x}\right) N \ell},
$$

where $f_{0}\left(\lambda_{e x}\right)$ is an intensive quantity representing the fluorescence intensity at $\ell=0$ for a unit density solution, thus not depending on $N$. Since the fluorescence is collected through a pathlength of $L \sim 2 \mathrm{~mm}$ (Figure 1), the SIFE leads to the measured intensity $F_{N}\left(\lambda_{f}\right)$ given by:

$$
F_{N}\left(\lambda_{f}\right)=F_{N}^{\prime}\left(\lambda_{f}\right) 10^{-\tilde{\sigma}\left(\lambda_{f}\right) N L},
$$

i.e.

$$
F_{N}\left(\lambda_{f}\right)=f_{0}\left(\lambda_{e x}\right) N 10^{-N\left[\tilde{\sigma}\left(\lambda_{e x}\right) \ell+\tilde{\sigma}\left(\lambda_{f}\right) L\right]}
$$

To eliminate $f_{0}\left(\lambda_{e x}\right)$, we assume that the fluorescence spectrum $F_{N_{0}}\left(\lambda_{e x}\right)$ corresponding to the QD density $N_{0}$ is known and can be considered as a reference spectrum:

$$
F_{N}\left(\lambda_{f}\right)=F_{N_{0}}\left(\lambda_{f}\right) \frac{N}{N_{0}} 10^{-\left(N-N_{0}\right)\left[\tilde{\sigma}\left(\lambda_{e x}\right) \ell+\tilde{\sigma}\left(\lambda_{f}\right) L\right]} .
$$

Even if we precisely determined $\tilde{\sigma}\left(\lambda_{e x}\right)$ for both QD520 and QD610, we also need to compute $\tilde{\sigma}\left(\lambda_{f}\right)$ for every $\lambda_{f}$. To do so, we use the absorbance spectra given in Figure $3 \mathrm{c}$ :

$$
\tilde{\sigma}\left(\lambda_{f}\right)=\frac{\mathscr{A}_{\mathrm{ref}}\left(\lambda_{f}\right)}{N_{\mathrm{ref}} \xi}
$$

where $\mathscr{A}_{\text {ref }}(\lambda)$ is the absorbance spectrum thus taken as reference and $N_{\text {ref }}$ the corresponding density. Here, the chosen reference absorbance spectra correspond to $N_{\text {ref }}=3.6610^{20} \mathrm{~m}^{-3}$ for QD520 and $N_{\text {ref }}=1.4710^{20} \mathrm{~m}^{-3}$ for QD610.

The results of this model are given in Figure 5. The fluorescence spectra of the lowest concentrations have been chosen as references (i.e. $F_{N_{0}}\left(\lambda_{f}\right)$ ) to model the fluorescence spectra of the highest concentrations: unlike the latter, the former are indeed supposed to be barely affected by PIFE and SIFE so that they constitute the most appropriate reference spectra for fluorescence. Moreover, since we cannot precisely measure the lengths $\ell$ and $L$, we consider them as fitting parameters. The two modeling curves presented in Figure $5 \mathrm{a}$ are obtained for the parameters given in Table 2. They show how accurate our computations are. Indeed, they account for the redshift of fluorescence spectra and
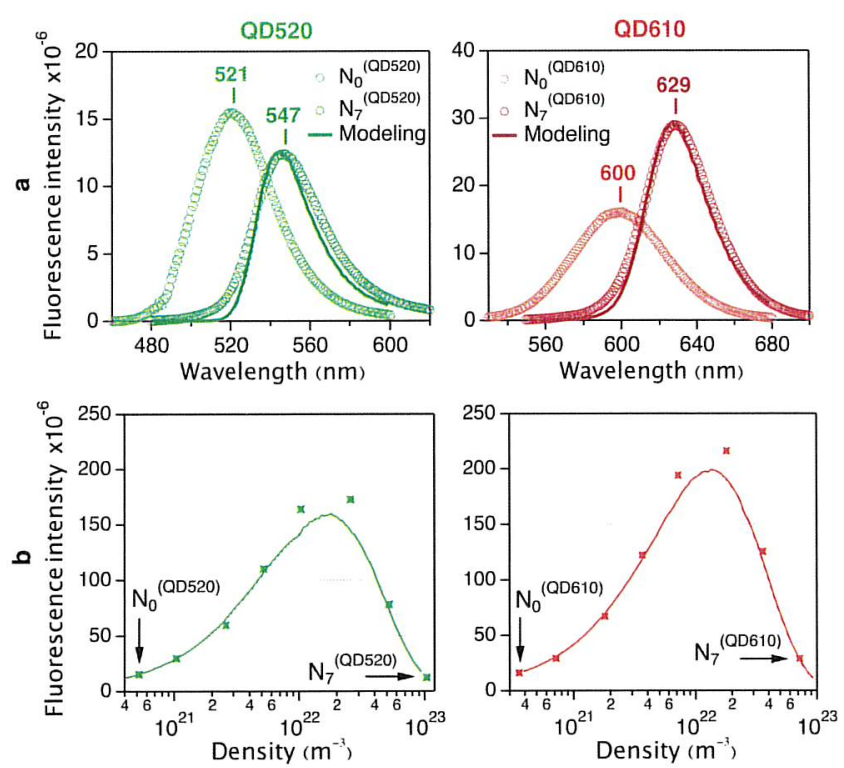

Fig. 5 (a) Fluorescence spectra of QD520 and QD610 at two different concentrations, denoted by $N_{0}$ and $N_{7}$. The circles correspond to the measurements and the solid lines to the model of Eq. (12). The modeling of $N_{7}^{(\mathrm{QD} 520)}$ and $N_{7}^{(\mathrm{QD} 610)}$ is obtained by taking $N_{0}^{(\mathrm{QD} 520)}$ and $N_{0}^{(\mathrm{QD} 610)}$ as reference spectra. (b) Modeling of the maximum of fluorescence intensity with respect to QD density. The dots correspond to the experimental data given in Fig. $4 a$.

\begin{tabular}{lll}
\hline & $\ell(\mathrm{mm})$ & $L(\mathrm{~mm})$ \\
\hline QD520 & 0.77 & 2.2 \\
QD610 & 0.85 & 1.5
\end{tabular}

Table 2 Fitting parameters $\ell$ and $L$. The two sets of parameters are not strictly equal because we slightly changed the position of the cuvette between the two sets of measurements to optimize the fluorescence signal.

the decreasing of their bandwidth. These two effects are direct consequences of SIFE. They come from the overlap between the absorbance and fluorescence spectra, clearly visible in Figure 3c: at high concentration, the left sides of the fluorescence spectra are cut off by the absorption, hence their redshift and the decreasing of their bandwidth. Furthermore, as depicted in Figure $5 b$, the maximum fluorescence intensity $F_{N}\left(\lambda_{f}^{\max }\right)$ is also well described by combining Equation (12) with Equations (5) and (6). In particular, we can extract from Equation (12) the exact expression of the critical density $N^{*}$ :

$$
\left.\frac{\partial F_{N}}{\partial N}\right|_{N^{*}}=0 \quad \Longrightarrow \quad N^{*}=\frac{1}{\tilde{\sigma}\left(\lambda_{e . x}\right) \ell+\tilde{\sigma}\left(\lambda_{f}\right) L},
$$

which generalizes the expression given in Ref. 30 and highlights the role of the geometric configuration through the two parameters $\ell$ and $L$. In the case of brighter QDs (with greater absorption cross sections), we notice that $N^{*}$ would decrease: the more QDs absorb light, the smaller the critical density is, and the stronger inner filter effects are. Equation (14) thus supports our understanding of the phenomenon. As a result, PIFE and SIFE completely account for the concentration dependent behaviour of QDs fluores- 
cence. It then does not prove necessary to describe the decreasing of fluorescence at high concentration by collisional quenching. This mechanism is indeed often invoked. It is based on the assumption that a collision between two particles, of which one is a fluorophore in its excited state, can lead to the non-radiative relaxation of this latter ${ }^{31}$ so that its lifetime (the inverse quantity of the spectral bandwidth) and its quantum efficiency (to which the fluorescence intensity is proportional) decrease. In our case, QDs could be their own quenchers. Since the probability of a twoparticle collision is proportional to the particle density, we could attempt to account for the decreasing of the QDs fluorescence intensity at high concentration in virtue of such a mechanism. But the Stern-Volmer equation that theoretically describes collisional quenching indicates that the spectral bandwidth $\gamma$ is related to the particle density $N$ by Equation (15) ${ }^{31}$ :

$$
\gamma=\gamma_{0}+k_{q} N
$$

where $\gamma_{0}$ is the spectral bandwidth in absence of collision, and $k_{q}>0$ the two-particle quenching constant. Incidentally, if the present concentration dependent behaviour of QDs was linked to collisional quenching, the bandwidth of their fluorescence would be expected to increase. But fluorescence spectroscopy shows a decreasing of the bandwidth that the implementation of inner filter effects fits very well. Therefore, the intrinsic properties of QDs are not affected by their concentration: individually, they preserve their optical and then quantum properties.

Last but not least, this study also provides a simple and flexible procedure to handle fluorescence spectra in the case of highly concentrated samples. A generalization of the method is summarized in $\mathrm{ESI}^{\dagger}$ for right angle, front-face and collinear configurations (Sections D and E), itemizing all the steps of computation so that everyone can apply it for their own setup and experiment.

\section{Conclusion}

We have demonstrated the relevance of inner filter effects to explain both the decreasing of fluorescence at high concentration and its spectral redshift. Contrary to the explanations often put forward, this decreasing is mainly due to the competition between fluorescence and absorption: we do not need to invoke collisional quenching to account for these observations.

Furthermore, regarding the concentration dependent redshift of fluorescence, it would be tempting to consider it universal. Though, this redshift is a direct consequence of SIFE and thus depends on both the geometry of the system (with $\ell$ and $L$ ) and the presence of other chromophores, so that the relations which give $\lambda_{f}^{\max }$ as a function of the QD density cannot be used to deduce the QD concentration from the measurement of $\lambda_{f}^{\max }$ in an unknown solution. Contrary to what is commonly asserted, it is impossible to provide any general calibration curve operable for fluorescence biosensing. Instead, our method can be adapted to every configuration and every fluorophore, provided the absorbances of all the present chromophores are known.

\section{Conflicts of interest}

There are no conflicts of interest to declare.

\section{Acknowledgements}

Research leading to these results has received funding from the International Scientific Cooperation Program of the CNRS (Centre National de la Recherche Scientifique) under Grant agreement number PICS07339. The authors acknowledge Research Engineer P. Beaunier (UPMC Univ Paris 06, Sorbonne Univ, CNRS, UMR 7197, Lab React Surface, 75005 Paris, France) for her precious help in the realization and interpretation of HR-TEM measurements. We thank Dr. B. Busson for fruitful scientific discussion.

\section{References}

1 L. E. Brus, J. Chem. Phys., 1984, 80, 4403-4409.

2 A. P. Alivisatos, A. L. Harris, N. J. Levinos, M. L. Steigerwald and L. E. Brus, J. Chem. Phys., 1988, 89, 4001-4011.

3 M. G. Bawendi, M. L. Steigerwald and L. E. Brus, Annu. Rev. Phys. Chem., 1990, 41, 477-496.

4 R. R. Cooney, S. L. Sewall, D. M. Sagar and P. Kambhampati, Phys. Rev. Lett., 2009, 102, 127404.

5 J. Nanda, S. A. Ivanov, M. Achermann, I. Bezel, A. Piryatinski and V. I. Klimov, J. Phys. Chem. C, 2007, 111, 15382-15390.

6 N. Somaschi, V. Giesz, L. D. Santis, J. C. Loredo, M. P. Almeida, G. Hornecker, S. L. Portalupi, T. Grange, C. Antón, J. Demory, C. Gómez, I. Sagnes, N. D. Lanzillotti-Kimura, A. Lemaître, A. Auffeves, A. G. White, L. Lanco and P. Senellart, J. Phys. Chem. C, 2016, 10, 340-345.

7 A. A. Umar, A. H. Reshak, M. Oyama and K. J. Plucinski, J. Mater. Sci. Mater: Electron., 2012, 23, 546-550.

8 C. Humbert, A. Dahi, L. Dalstein, B. Busson, M. Lismont, P. Colson and L. Dreesen, Journal of Colloid and Interface Science, 2015, 445, 69-75.

9 M. Law, M. C. Beard, S. Choi, J. M. Luther, M. C. Hanna and A. J. Nozik, Nano Lett., 2008, 8, 3904-3910.

10 D. Vanmaekelbergh and P. Liljeroth, Chem. Soc. Rev., 2005, 34, 299-312.

11 R. F. Heuff, J. L. Swift and D. T. Cramb, Phys. Chem. Chem. Phys., 2007, 9, 1870-1880.

12 J. M. Costa-Fernandez, R. Pereiro and A. Sanz-Medel, Trends in Analytical Chemistry, 2006, 25, 207-218.

13 C. M. Tyrakowski and P. T. Snee, Phys. Chem. Chem. Phys., 2014, 16, 837-855.

14 K. D. Wegner and N. Hildebrandt, Chem. Soc. Rev., 2015, 44, 4792-4834.

15 K.-T. Yong, W.-C. Law, I. Roy, Z. Jing, H. Huang, M. T. Swihart and P. N. Prasad, J. Biophotonics, 2011, 4, 9-20.

16 F.-C. Chien, C. W. Kuo and P. Chen, Analyst, 2011, 136, 1608 1613.

17 J. Li and J.-J. Zhu, Analyst, 2013, 138, 2506-2515. 
18 F. M. Raymo and I. Yildiz, Phys. Chem. Chem. Phys., 2007, 9, 2036-2043.

19 D. Segets, J. M. Lucas, R. N. K. Taylor, M. Scheele, H. Zheng, A. P. Alivisatos and W. Peukert, ACS Nano, 2012, 6, 90219032.

20 Y. Kobayashi, T. Nishimura, H. Yamaguchi and N. Tamai, $J$. Phys. Chem. Lett., 2011, 2, 1051-1055.

21 B. Zorman, M. V. Ramakrishna and R. A. Friesner, J. Phys. Chem., 1995, 99, 7649-7653.

22 I.-S. Liu, H.-H. Lo, C.-T. Chien, Y.-Y. Lin, C.-W. Chen, Y.-F. Chen, W.-F. Su and S.-C. Liou, J. Mater. Chem., 2008, 18, 675682.

23 Y. Masumoto and K. Sonobe, Phys. Rev. B, 1997, 56, 9734 9737.

24 P. Dagtepe, V. Chikan, J. Jasinski and V. J. Leppert, J. Phys. Chem. C, 2007, 111, 14977-14983.

25 A. C. Vinayaka and M. S. Thakur, Luminescence, 2013, 28, 827-835.

26 S. F. Wuister, I. Swart, F. van Driel, S. G. Hickey and C. de Mello Donegá, Nano Lett., 2003, 3, 503-507.

27 M. Jin, Y.-Huang and J.-X. Luo, Spectroscopy and Spectral Analysis, 2015, 35, 420-423.

28 B. S. Rinehart and C. G. L. Cao, Opt. Eng., 2016, 55, 87106.

29 O. Divya and A. K. Mishra, Analytica Chimica Acta, 2008, 630, 47-56.

30 K. Pawlak, A. Skrzypczak and G. E. Bialek-Bylka, Inner Filter Effect in the Fluorescence Emission Spectra of Room Temperature Ionic Liquids with- $\beta$-Carotene, in Applications of Ionic Liquids in Science and Technology, InTech, 2011.

31 J. R. Lakowicz, Quenching of Fluorescence, in Principles of Fluorescence Spectroscopy, Springer, 3rd edn, 2006.

32 Sigma-Aldrich, 777935 CdTe core-type quantum dots, http://www.sigmaaldrich.com/catalog/product/ aldrich/777935, (accessed February 2017).

33 Sigma-Aldrich, 777951 CdTe core-type quantum dots, http://www.sigmaaldrich.com/catalog/product/ aldrich/777951, (accessed February 2017).

34 S. Sapra and D. D. Sarma, Phys. Rev. B, 2004, 69, 125304.

35 C. de Mello Donegá and R. Koole, J. Phys. Chem. C, 2009, $113,6511$.

36 E. Groeneveld, C. Delerue, G. Allan, Y.-M. Niquet and C. de Mello Donegá, J. Phys. Chem. C, 2012, 116, 23160.

37 Z. M. Nassar, M. H. Yükselici and A. A. Bozkurt, Phys. Status Solidi, 2016, 253, 1104.

38 A. Samanta, Z. Deng and Y. Liu, Langmuir, 2012, 28, 8205.

\section{Table of Contents}

A modeling of quantum dots' fluorescence spectra altered by inner filter effects is applicable to any type of fluorophores.

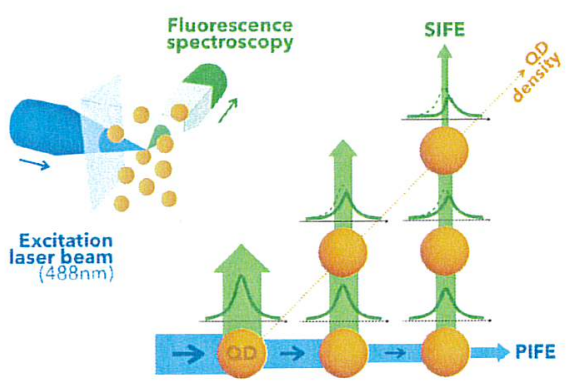

